\title{
Lorentz Force Flowmeter for Liquid Aluminum: Laboratory Experiments and Plant Tests
}

\author{
YURII KOLESNIKOV, CHRISTIAN KARCHER, and ANDRÉ THESS
}

\begin{abstract}
This article aims to demonstrate that molten metal flow at a high temperature can be measured effectively in a contactless manner by using external direct current magnetic fields. The device applied in the present work is termed Lorentz force flowmeter (LFF) and is based on exposing the flow to a magnet system and measuring the drag force acting on it. Two series of measurements are reported. In the first series, we perform a model experiment in the laboratory using the eutectic alloy GaInSn, which is liquid at room temperature. The second series of measurements is devoted to two plant tests on flow measurement of a liquid aluminum alloy. In both tests, the force acting on the magnet system is measured that is equal to the Lorentz force acting on the flow. To generalize our results, we also derive the scaling law that relates the force acting on a localized magnet system to the flow rate of a fluid with arbitrary electrical conductivity. This law shows that LFF, if properly designed, has a wide range of potential applications in ferrous and nonferrous metallurgy.
\end{abstract}

DOI: $10.1007 / \mathrm{s} 11663-011-9477-6$

(C) The Author(s) 2011. This article is published with open access at Springerlink.com

\section{INTRODUCTION}

THIS present work is devoted to demonstrating the applicability of a recently developed electromagnetic noncontact flow measurement device - the Lorentz force flowmeter (LFF) - to flow measurement in the production of secondary aluminum.

A considerable part of the world's aluminum demand is satisfied through secondary aluminum production. ${ }^{[1,2]}$ During the production process, aluminum scrap is molten in special rotary furnaces fired by heavy oil to support a sufficiently high temperature. The burner system generates temperatures higher than $1973 \mathrm{~K}$ $\left(1700^{\circ} \mathrm{C}\right)$ inside the furnace. The capacity of the furnaces shows a typical range of 8 to 14 tons. After tapping the furnace, the primary melt flows under the action of gravity through an open channel into converters that are located below ground level. In these converters, the final composition of the alloy is prepared by adding alloying elements including $\mathrm{Si}, \mathrm{Cu}, \mathrm{Fe}, \mathrm{Mn}$, and $\mathrm{Cr}$ among others. Finally, after tapping of the converter, the final melt flows again through an open channel either to the casting machine, where it will solidify, or into a preheated crucible, which is used to deliver the liquid aluminum to the customer. ${ }^{[1-3]}$

To monitor and control the production process, it would be desirable to measure the mean velocity of the liquid aluminum continuously to deduce the mass-flux and volume-flux. Until now, however, no melt-flow

YURII KOLESNIKOV, Head of Research Group, CHRISTIAN KARCHER, Head of Research Group, and ANDRE THESS, Head of Department, are with the Institute of Thermodynamics and Fluid Mechanics, Department of Mechanical Engineering, Ilmenau University of Technology, 98684 Ilmenau, Germany. Contact e-mail: yuri.kolesnikov@tu-ilmenau.de

Manuscript submitted May 20, 2010.

Article published online February 24, 2011. measurement system is commercially available. In general, the production process is controlled only by weighing the scrap and either the solid blocks or the containers holding the final liquid melt (i.e., by taking data only at the beginning and the end of the process). During the production, melting salt $(\mathrm{NaCl}+\mathrm{KCl})$ is added to avoid heat waste and to absorb the burn-off resulting from scrap contamination with plastics, oil, and dirt. So, after tapping, it is not known how much aluminum remains in the rotary furnace and/or how much aluminum is in the converter. ${ }^{[3]}$ Therefore, important information during the production process is not available. For instance, the scrap performance cannot be evaluated exactly and the exact amount of additions into the converter cannot be calculated.

The object of the present study is the development and demonstration of the feasibility of a noncontact electromagnetic system to measure a liquid metal flow. Our approach embodies the Lorentz force velocimetry ${ }^{[4-10]}$ technique, which is physically based on measuring the force acting on an external magnet system that interacts with the flow. This force is exactly equal to the braking Lorentz force induced in a flowing conducting fluid by an external magnetic field. In what follows, a measurement system based on the Lorentz force velocimetry principle will be called LFF.

As will be detailed in the following, the LFF possesses several advantages that make it attractive to measure flows in hot and aggressive melts. First, the method is entirely contactless. Second, the relation between the measured force and the desired volumetric flow rate is often linear. Third, the slope of this linear function - the calibration coefficient - is independent of the viscosity and density of the liquid metal and depends only on the magnetic field magnitude, the electrical conductivity of the liquid metal, and the geometry of the channel in which the liquid metal flows. Therefore, a calibration 
curve obtained for one liquid metal can be used directly for other liquid metals provided that their electrical conductivities are known. In aluminum production, for instance, numerous standard alloys exist that have welldefined conductivities for which the calibration factors thus can be tabulated.

In what follows, namely in Section II, we give a brief review of the principles on which a LFF is based. In Section III, we explain how the measured force signal of an LFF can be converted into the desired volume flux. In Section IV, which is the main body of the present work, we report the results of velocity measurements both in a laboratory, room-temperature liquid metal flow using a small-scale LFF and in the plant of a producer of secondary aluminum using a full-scale LFF prototype for industrial flows. In Section $\mathrm{V}$ we summarize our conclusions and indicate future research directions.

\section{MEASUREMENT PRINCIPLE}

The LFF is based on the observation that a Lorentz force is induced within an electrically conducting melt (e.g., a liquid metal that is moving across magnetic field lines as shown in Figure 1(a)). The Lorentz force acting on the liquid metal is pointing in the opposite direction of the flow and is proportional to the volumetric flow rate of the melt. ${ }^{[4,5]}$ This is the well-known eddy current braking effect used in a couple metallurgical processes like continuous casting of steel. ${ }^{[11]}$ However, when an electrically conducting fluid flows across an externally arranged magnet system, then by Newton's third law, a force of exactly the same magnitude as the mentioned Lorentz force but pointing in the opposite direction is acting on this magnet system. This force is directly proportional to the flow rate of the melt and can be measured exactly. Although the described phenomenon occurs whether the magnetic field is generated by a heavy electromagnet or by a lightweight permanent

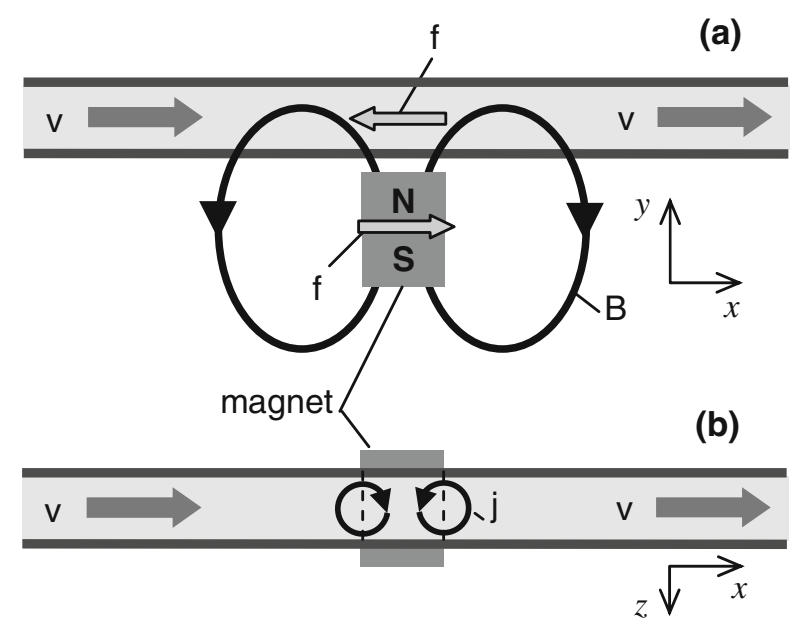

Fig. 1-Principle sketch of LFF showing the interaction of the flow of an electrically conducting fluid with the magnetic field. (a) Side view, (b) top view; $\mathrm{v}$ is flow velocity, $\mathrm{B}$ is magnetic field lines, $\mathrm{f}$ is Lorentz force, and $\mathrm{j}$ is induced electrical eddy currents. magnet, it is only because of the recent advent of powerful rare earth permanent magnets ${ }^{[12,13]}$ and design tools for permanent magnet systems that a practical realization of this principle has now become possible. ${ }^{[5]}$

The principle of an LFF can be understood better if one derives the basic scaling law of this device by invoking Ohm's law of a moving electrically conducting material in the following form:

$$
\mathbf{j}=\sigma(\mathbf{E}+\mathbf{v} \times \mathbf{B})
$$

where $\mathbf{j}$ is the electric current density, $\sigma$ is the electrical conductivity of fluid, $\mathbf{E}$ is the electric, $\mathbf{B}$ is the magnetic fields in the fluid, and $\mathbf{v}$ is the fluid velocity. An order-of-magnitude estimate based on this law readily shows that the component of the Lorentz force along the direction of the mean flow can be estimated as follows:

$$
f=(\mathbf{j} \times \mathbf{B})_{x} \sim \sigma v B^{2}
$$

Equation [2] shows that the force density is proportional to the square of the amplitude of the magnetic field, which indicates that the construction of lightweight high-field magnet systems is of crucial importance for designing LFF with high sensitivity. The electric eddy currents in the fluid induced by the external magnetic field are shown qualitatively in Figure 1(b). The phenomena sketched in Figures 1(a) and (b) are well known ${ }^{[14]}$ and have found a variety of applications for flow control in metallurgy. ${ }^{[10,11]}$ Their application to electromagnetic flow measurement using LFF consists of measuring the force acting on a magnet system and deducing the unknown flow velocity or volume flux from this force measurement.

\section{CALIBRATION}

The key question for a successful implementation of LFF is how the unknown flow rate of a liquid metal can be computed from the measured force. This question can be answered either by performing a numerical simulation of the full magnetohydrodynamic problem or by calibration of the measuring system. In the present work, we are concerned exclusively with the second method.

\section{A. Lorentz Force Measurement}

The basic calibration equation for an LFF is expressed as follows:

$$
F=c \sigma\left(T_{\mathrm{L}}\right) B^{2}\left(T_{\mathrm{M}}\right) q
$$

where $F$ is the measured Lorentz force and $q$ is the unknown volumetric flow rate. $B$ is the amplitude of the magnetic field at an arbitrary fixed point in the liquid metal, $T_{\mathrm{M}}$ is the temperature of the magnet system, and $c$ is a calibration factor that depends on the geometry of the channel as well as on the spatial structure of the magnetic field. In this equation, which is derived from Eq. [2], the force is in Newton (N). In the general case, 
the value of the electric conductivity $\sigma$ depends on the temperature $T_{\mathrm{L}}$ of the liquid metal. Measurements of $\sigma\left(T_{\mathrm{L}}\right)$ in molten aluminum alloys (e.g., AlSi12) ${ }^{[15]}$ show that this quantity varies by approximately 4.5 pet when the temperature varies in the range $973 \mathrm{~K}$ to $1073 \mathrm{~K}$ $\left(700{ }^{\circ} \mathrm{C}\right.$ to $\left.800{ }^{\circ} \mathrm{C}\right)$. However, during the transport processes described in the introduction, the variation of the temperature is usually smaller and confined to the range $1023 \mathrm{~K}$ to $1053 \mathrm{~K}\left(750{ }^{\circ} \mathrm{C}\right.$ to $\left.780{ }^{\circ} \mathrm{C}\right)$; therefore, it follows that within the stated limits of operating temperature, the conductivity $\sigma$ can be variable within 1.25 pct. If the temperature is permanently measured, which is often the case in production processes, then the variation of conductivity can be accounted for by introducing temperature dependence for conductivity into the measurement software. In industrial conditions, the magnet system of the LFF is greater than room temperature because the system is situated in the immediate vicinity of the channel in which the hightemperature liquid metal flows.

In our LFF prototypes, we use commercial rareearth permanent magnets consisting of $\mathrm{NdFeB}$. To characterize the dependence of their magnetization on temperature, we carried out a preliminary experiment in which a single block was exposed to different temperatures. We found that the magnetic induction decreased by 8.5 pct when the temperature of the magnets was increased from $293 \mathrm{~K}$ to $363 \mathrm{~K}\left(20^{\circ} \mathrm{C}\right.$ to $\left.90{ }^{\circ} \mathrm{C}\right)$. The experiment was carried out with a magnet that has dimensions of $30 \times 20 \times 100 \mathrm{~mm}$. An indication of the temperature was produced by a standard thermoresistor placed on the magnet surface. The temperature was registered by a multivoltmeter. For an accurate calibration, it is necessary to introduce a field dependence on temperature into the force calculation program covered by Eq. [3]. In particular, the dependence is calculated according to the following:

$$
B\left(T_{\mathrm{M}}\right)=B_{0}\left[1-\alpha\left(T_{\mathrm{M}}-T_{0 \mathrm{M}}\right)\right]
$$

( $B_{0}$ is the magnetic field induction at the initial magnet temperature $\left.T_{0 \mathrm{M}}=293 \mathrm{~K}\left(20^{\circ} \mathrm{C}\right)\right)$. As follows from our preliminary experiment, the coefficient of temperature variation of the magnetic field is $\alpha=1.116 \times 10^{-3}$ $\left(\mathrm{K}^{-1}\right)$.

An industrial channel for liquid aluminum transportation on the external wall surface has a real temperature that does not exceed $323 \mathrm{~K}\left(50{ }^{\circ} \mathrm{C}\right)$. This is a consequence of the large thickness of 60 to $80 \mathrm{~mm}$ of the walls made of concrete, of their comparatively low thermal conductivity of about $0.5 \mathrm{~W} / \mathrm{m} \mathrm{K}$, and of natural air convection around the channel. Taking this into account, we are led to the conclusions that the magnetic induction deviation does not exceed 1 to 1.5 pct. Nonetheless, to monitor a possible temperature variation in the industrial tests, we install a thermocouple on the surface of magnet system.

The relationship for the calibration constant $c$ results from Eq. [3] as follows:

$$
c=\frac{F(q)}{\sigma B_{0}^{2} q}
$$

The dimension of $c$ is meter. Physically, $c$ can be interpreted as a lengthscale characterizing the magnetic field nonhomogeneity along the flow direction. The calibration constant for a given LFF can be determined either by a numerical simulation or experiments in which the flow rate $q$ is known. In this article, we determine $c$ experimentally both for laboratory and industrial LFFs.

To determine $c$ for our laboratory LFF described in Section IV-A, we measure the Lorentz force $F$ directly for different volumetric flow rates $q$ that are set up in a room-temperature, liquid-metal test channel. We choose the magnetic field induction $B_{0}$ equal to its value on the flow axis. In Section IV-B, for the calibration of our industrial LFF, we use one of two tests at aluminum production in a plant in which the total mass of discharged aluminum is known. In this case, we also measure Lorentz force and use the cumulative weight of aluminum in the end of the process to compute its flow rate through the channel.

\section{B. Volumetric Flow Rate and Cumulative Volume}

Once the electrical conductivity is known and the calibration constant $c$ has been determined, the timedependent volumetric flow rate $q(t)$ can be determined from the measured signals from the force-sensor $F(t)$ as well as the measured temperature of the magnet $T_{\mathrm{M}}(t)$ by applying Eq. [3] as follows:

$$
q(t)=\frac{F(t)}{c \sigma B_{0}^{2}\left[1-1.116 \times 10^{-3}\left(T_{\mathrm{M}}(t)-T_{0 \mathrm{M}}\right)\right]^{2}}
$$

From the volumetric flow rate in a time span from $t=t_{0}$ to $t=t_{1}$, one can compute the cumulative liquid metal volume as follows:

$$
V=\int_{t_{0}}^{t_{1}} q(t) d \tau
$$

\section{Mass Flow Rate and Cumulative Mass}

Combining Eq. [6] with the fluid density yields the following mass flow rate:

$$
m(t)=\rho q(t)
$$

From Eq. [7], the comulative mass within a given period of time is determined as follows;

$$
M=\rho \int_{t_{0}}^{t_{1}} q(t) d \tau
$$

\section{Drag Coefficient}

Because of the applied magnetic field, a drag force (Lorentz force) occurs in the gap between the LFF 
magnet poles. Depending on the magnetohydrodynamic parameters, this force can exert some influence on the flow of the liquid metal. To estimate the corresponding drag coefficient, we suppose that an induced potential difference $v B h$ (where $v$ is flow velocity and $h$ is a channel width in direction perpendicular to the magnetic field) generates the eddy electric currents as shown in Figure 1(b) and follows from Eq. [1]. A pressure difference $\Delta p=k_{0} \sigma v B^{2} h$ is then associated with these currents (Eq. [2]). The factor $k_{0}$ depends on the spatial decay of the magnetic field ${ }^{[14]}$ and is estimated as $k_{0}=0.1$. Using the general formula for drag coefficient, we obtain the following:

$$
\zeta=\frac{2 \Delta p}{\rho v^{2}} \approx 0.2 \frac{\sigma B^{2} h}{\rho v^{2}}=0.2 \mathrm{~N}
$$

where the nondimensional number $N$ is called the interaction parameter and represents the ratio of Lorentz and inertia forces. In the case $N>1$, one would have an appreciable pressure loss. ${ }^{[7]}$ As a result, in open channels, the free surface can be inclined within the magnetic field area, which can lead to a deterioration of measurement accuracy. For the industrial tests in our work the condition, $N<<1$ is always satisfied (Table II) so that the free surface of flows is not distorted. The case $N>1$ is acceptable for closed channels when a deformation of velocity profile does not influence the accuracy of measurement.

\section{LABORATORY AND INDUSTRIAL MEASUREMENTS}

Demonstrating LFF application for the feasibility of a measurement in liquid aluminum consists of two steps. The first step is a conduction of a reduced-scale laboratory experiment with liquid metal that has a low melting temperature to establish the calibration procedure. The working fluid used in this experiment is the eutectic alloy $\mathrm{Ga}^{68}{ }^{\mathrm{pct}} \mathrm{In}^{20}{ }^{\mathrm{pct}} \mathrm{Sn}^{12}$ pct, which is liquid at room temperature and has a similar electrical conductivity as the molten eutectic alloy of A1Si12. The second step of our work consists of full-scale plant tests with liquid aluminum alloy A1Si12 at operating temperature. The physical properties of both liquid metals are listed in Table I. For these properties, the characteristic parameters obtained in laboratory and industry are presented in Table II.

Table I. Physical Properties of Working Fluids

\begin{tabular}{|c|c|c|}
\hline Properties & $\begin{array}{c}\mathrm{Ga}^{68}{ }^{\mathrm{pct}} \mathrm{In}^{20} \mathrm{pct}^{\mathrm{S}} \mathrm{Sn}^{12 \mathrm{pct}} \\
T_{\mathrm{L}}=293 \mathrm{~K} \\
\left(20{ }^{\circ} \mathrm{C}\right)\end{array}$ & $\begin{array}{c}\text { AlSi12 } \\
T_{\mathrm{L}}=1053 \mathrm{~K} \\
\left(780^{\circ} \mathrm{C}\right)\end{array}$ \\
\hline $\begin{array}{l}\text { Mass density } \\
\rho\left(\mathrm{kg} / \mathrm{m}^{3}\right)\end{array}$ & 6363 & 2370 \\
\hline $\begin{array}{l}\text { Kinematic viscosity } \\
\qquad(\mathrm{m} / \mathrm{s})\end{array}$ & $3.40 \times 10^{-7}$ & $4.21 \times 10^{-7}$ \\
\hline $\begin{array}{l}\text { Electrical conductivity } \\
\sigma(\mathrm{Ohm} \mathrm{m})^{-1}\end{array}$ & $3.31 \times 10^{6}$ & $3.01 \times 10^{6}$ \\
\hline
\end{tabular}

\section{A. Laboratory Measurements}

The experiment is carried out with a laboratory LFF and mentioned working fluid $\mathrm{Ga}^{68}{ }^{\mathrm{pct}} \mathrm{In}^{20}{ }^{\mathrm{pct}} \mathrm{Sn}^{12}$ pct with the properties given in Table I. The aim of this experiment is to determine a procedure of calibration factor $c$ for a well-defined flow for which the flow rate can be measured and monitored accurately.

The laboratory model of the LFF consists of a magnetic system with two permanent magnets installed on an iron yoke as shown in Figure 2(a), of a mechanical system for force transmission (not shown), and of electronic commercial scales with a measuring accuracy of $10^{-4} \mathrm{~N}$. Both magnets are made of $\mathrm{NdFeB}$ and have dimensions of $30 \times 20 \times 100 \mathrm{~mm}$ and a magnetic field induction of $550 \mathrm{mT}$ on the surface with a size of $30 \times 100 \mathrm{~mm}$. The magnetic gap of the system is $35 \mathrm{~mm}$. The yoke of $20 \mathrm{~mm}$ in thickness provides an increase of magnetic field induction in the middle point of gap by 15 pct. The overall weight of the magnet system is $2.52 \mathrm{~kg}$. The experimental channel is placed into the gap of the magnet system from earlier.

The distributions of the magnetic field along the channel height and in the direction of flow are shown in Figures 2(a) and (b). In vertical direction $z$ within the height of channel, the nonhomogeneity of the field does

Table II. Governing Parameters for Experimental Studies of LFF in Laboratory and Industry (Maximum Values)

\begin{tabular}{lll}
\hline Mean Parameters & Laboratory & \multicolumn{1}{c}{ Industry } \\
\hline Lorentz force $F(\mathrm{~N})$ & 10 & 0.5 \\
Magnetic field induction & 425 & 13.5 \\
$\quad B_{0}(\mathrm{mT})$ & & \\
Reynolds number Re & $0.185 \times 10^{5}$ & $2.38 \times 10^{5}$ \\
Interaction parameter $N$ & 1.32 & 0.12 \\
Volumetric flow rate $q\left(\mathrm{~m}^{3} / \mathrm{s}\right)$ & $0.5 \times 10^{-3}$ & $10.55 \times 10^{-3}$ \\
Mass flow rate $m(\mathrm{~kg} / \mathrm{s})$ & 3.18 & 25.02 \\
Accumulated mass $M(\mathrm{~kg})$ & - & 8930 \\
Calibration factor & $1.605 \times 10^{-2}$ & $4.57 \times 10^{-2}$ \\
$\quad$ of LFV $c(\mathrm{~m})$ & & \\
\hline
\end{tabular}

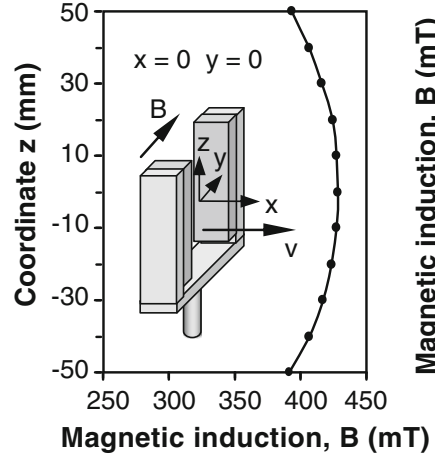

(a)

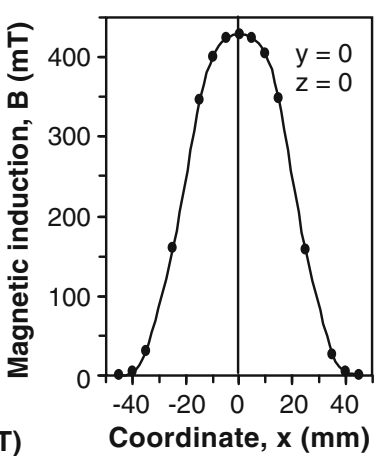

(b)
Fig. 2-View of magnet system and its magnetic field variations $(a)$ in vertical direction and $(b)$ along the direction of the mean flow; $v$ denotes the flow velocity; $B$ denotes the induction of the magnetic field. At the magnet surface, the magnetic field induction reaches $550 \mathrm{mT}$. 
not exceed 2.5 pct, whereas nonhomogeneity of field in flow direction $x$ is strong. High nonhomogeneity along the flow is favorable to the production of intensive electric currents $j$ as shown in Figure 1(b) and, consequently, to the generation of a large Lorentz force and the same force acting on magnet system.

The mechanical part of the LFF is a vertical metallic rod $500 \mathrm{~mm}$ in length linked with the magnetic system in its upper end and fixed in its middle point on a horizontal rotational axis. The point in which the force acts on the magnet system is at a distance of $305 \mathrm{~mm}$ from the rotational axis. The other end of the rod is linked with a balance weight for a stable equilibrium of the system at the initial instant of time of measurement. An additional horizontal rod of $175 \mathrm{~mm}$ in length is connected to the main vertical rod on the axis, and the other end is attached to the scales. The balance weight is chosen so that the imbalance of the mechanical system in the absence of measured force does not exceed $10^{-2} \mathrm{~N}$. This provides a high accuracy of force measurement because in a range of forces up to $10 \mathrm{~N}$ acting on the magnet system a deformation of scales sensor is less than $100 \mu \mathrm{m}$ (i.e., imbalance of mechanical system at this deformation remains practically previous).

A sketch of the whole experimental setup is presented in Figure 3(a), where the closed liquid metal loop 1 made of Plexiglas with a constant cross section of $h \times d=80 \times 10 \mathrm{~mm}$ is shown. The whole loop has a

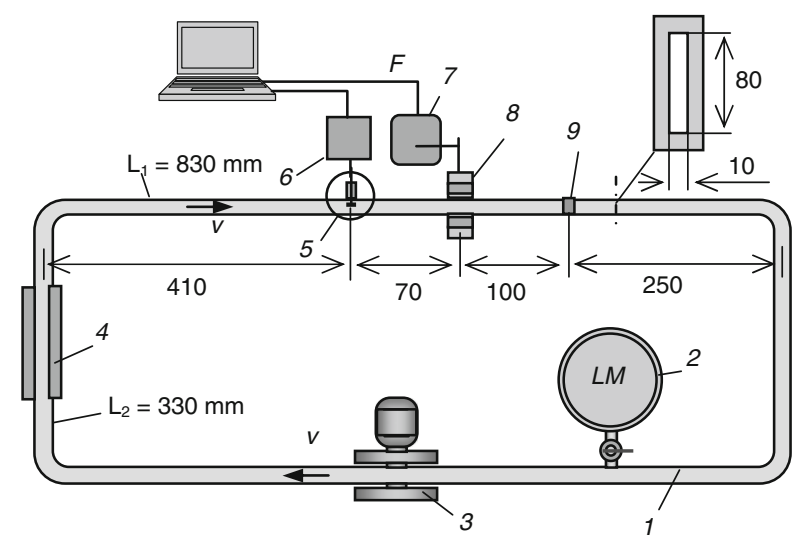

(a)

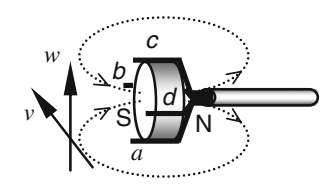

(b)

Fig. 3-(a) Diagram of liquid-metal laboratory experiment: 1 is liquid metal loop, 2 is the tank for liquid metal eutectic $\mathrm{Ga}^{68}$ pct $\mathrm{In}^{20} \mathrm{pct}^{\mathrm{Pn}} \mathrm{Sn}^{12 \mathrm{pct}}, 3$ is the electromagnetic pump, 4 is the heat exchanger, 5 is the Vivès probe with permanent magnet and four electrodes, 6 is the multivoltmeter 2700 DMM (Keithley Instruments, Inc., Cleveland, $\mathrm{OH}), 7$ is the electronic scales for Lorentz force $\mathrm{F}$ measurement, 8 is the LFF prototype, 9 is the controlling gate and window for installation of UVP sensor; $(b)$ Design of Vivès probe 5 , where $a, c$ and $b, d$ denote two pairs of electrodes measuring two components of flow in the local magnetic field generated by the cylindrical permanent magnet with the $\mathrm{N}$ and $\mathrm{S}$ poles. length $L_{1}=830 \mathrm{~mm}$ and a width $L_{2}=330 \mathrm{~mm}$. The total volume of the loop is $1860 \mathrm{~cm}^{3}$. The loop is filled by the eutectic alloy from a tank (2). An electromagnetic disk pump consisting of rotating permanent magnets (3) develops a pressure up to $1.0 \mathrm{bar}$ and a volumetric flow rate of $1.0 \mathrm{~L} / \mathrm{s}$. An electronic controller for the pump motor allows a reverse pumping in the loop and registration of pump rotation speed to bring these data into accord with mean velocity. To prevent heating of the liquid metal by the pump, a cooling system is connected to the short branches $\left(L_{2}=330 \mathrm{~mm}\right)$ of the loop. Cooling water passes outside over these walls, which are made of copper.

A Vivès probe (5; Figure 3(a)) is installed in the middle point of the channel cross section at a distance of $410 \mathrm{~mm}$ from the cross-section inlet. A signal from the Vivès probe is fed into a multivoltmeter 2700 DMM (Keithley Instruments, Inc., Cleveland, $\mathrm{OH}$ ) (6) and then to a computer for mean velocity calculation.

The signal of electronic scales (7) measuring a force on the magnet system is fed into the computer. The LFF prototype (8) is installed at a distance of $70 \mathrm{~mm}$ downstream from the Vivès probe (5). To examine the flow shape influence on readings of the LFF, we use a controlling gate (9), which is an obstacle partly blocking the cross section.

We apply the Vivès method ${ }^{[16]}$ for a definition of the mean velocity to find the volumetric flow rate $q$ shown in Eq. [3]. We reach this definition by measuring an averaged velocity $v$. To use this velocity for a definition of mean velocity, we check the homogeneity of the velocity profile over the cross section using an ultrasonic velocity profile (UVP) method. ${ }^{[17]}$ For such an examination, an UVP sensor of 8-mm diameter is installed in the hole (9; Figure 3) from earlier onto the upper surface at a distance of $250 \mathrm{~mm}$ from the end of the test-section length $L_{1}$. The measurements show that the vertical nonhomogeneity of the velocity profile is as small as $5 \mathrm{pct}$ for both direct and reverse flows. The design of the Vivès probe (Figure 3(b)) is a combination of a small cylindrical permanent magnet of 4-mm diameter and length as well as four copper potential electrodes $a, b, c$, and $d$ of $0.25 \mathrm{~mm}$ in diameter that measure two velocity components described by the following Eqs. [11] and [12]:

$$
\begin{gathered}
v=\frac{\Delta \varphi_{\mathrm{ac}}}{B l_{\mathrm{ac}}} \\
w=\frac{\Delta \varphi_{\mathrm{bd}}}{B l_{\mathrm{bd}}}
\end{gathered}
$$

where $\Delta \varphi_{\mathrm{ac}}$ and $\Delta \varphi_{\mathrm{bd}}$ are the electrical potential differences between sensitive points of electrodes, $l_{\mathrm{ac}}$ and $l_{\mathrm{bd}}$ are the distances between them, and $B$ is the magnetic field induction generated by the permanent magnet. These relationships result from Ohm's law (Eq. [1]). Of the components, $v$ coincides with the mean flow, and the other component $w$ is parallel to the vertical direction. Equations [11] and [12] may be simplified as follows:

$$
v=c_{v} \Delta \varphi_{\mathrm{ac}}
$$




$$
w=c_{w} \Delta \varphi_{\mathrm{bd}}
$$

where $c_{v}$ and $c_{w}$ are calibration factors. These factors take into account the intensity and configuration of the magnetic field as well as distances between electrodes. For probe calibration, we use a flow created by a rotating annular channel of $415-\mathrm{mm}$ diameter filled by the same eutectic alloy as in the experiment. At different rotation speeds, the probe immersed in the alloy gives linear dependences Eqs. [13] and [14] for corresponding components obtained by a probe orientation to the flow (Figure 3(b)). Calibration shows that the factors $c_{v}$ and $c_{w}$ are $7.48 \times 10^{3}(\mathrm{~m} / \mathrm{s} \mathrm{V})$ and $3.35 \times 10^{3}(\mathrm{~m} / \mathrm{s} \mathrm{V})$, respectively, if the potential difference is taken in volts and velocity components in $\mathrm{m} / \mathrm{s}$. Measurements by the probe in the test-section of the loop shows that the $w$ component is 50 -fold smaller than the $v$ component. To define the volumetric flow rate, the signal $\Delta \varphi_{\text {ac }}$ passing through the voltmeter is applied to a computer in which, according to Eq. [13] and knowledge of the area of flow cross section, the flow rate $q=v \times A$ (where $A$ denotes the area of flow cross section and $v$ denotes mean velocity) is computed. For instance, the electrical signal $\Delta \varphi_{\mathrm{ac}}=10.1 \times 10^{-5}$ volts corresponds to an average maximum velocity of $\left(v_{a}\right)_{\max }=0.755 \mathrm{~m} / \mathrm{s}$. With regard to the realization of a turbulent regime in the experiment (Table II), a mean velocity is $v=$ $0.625 \mathrm{~m} / \mathrm{s} \quad\left(\left(v_{a}\right)_{\max }=1.21 \times v\right.$ for turbulent regime, and calculating the flow rate gives $q=5 \times 10^{-4} \mathrm{~m}^{3} / \mathrm{s}$ $(0.5 \mathrm{~L} / \mathrm{s})$. A similar measurement in the GaInSn alloy flow is described in a recent article. ${ }^{[18]}$

To investigate the influence of the flow profile on the flow rate measurement, we obstruct the cross section to 0 pct, 50 pct, and 75 pct by inserting a solid obstacle that blocks the cross section while keeping the flow rate constant, as shown in Figure 4. In contrast to the present work, in Reference 5, a LFF has been used with two pairs of similar magnets separated by $50 \mathrm{~mm}$ from

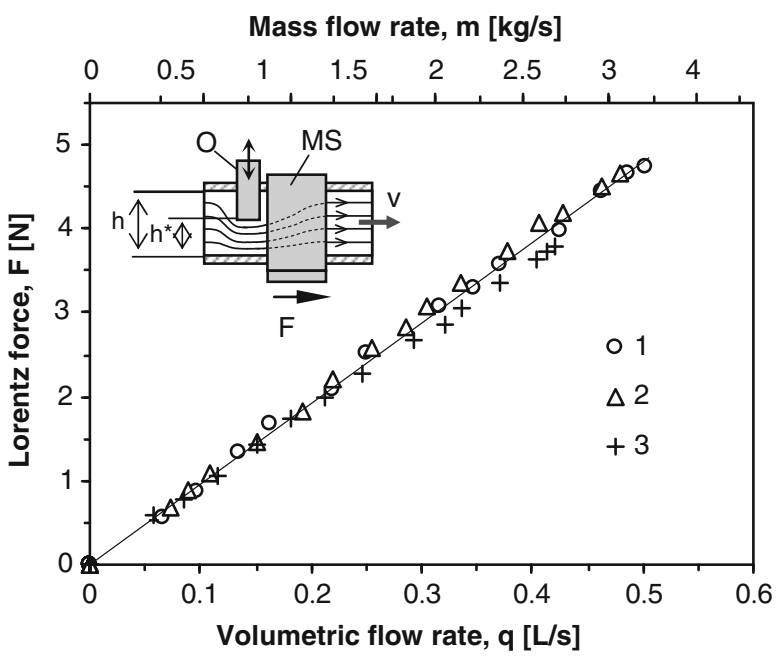

Fig. 4-Lorentz force as a function of volumetric and mass flow rates through the cross section of channel at different flow blocking levels of obstruction: 0 pct for curve 1,50 pct for curve 2, and 75 pct for curve 3 . In the sketch, MS is the magnet system, $\mathrm{O}$ is the solid obstruction commensurable with the channel width, and arrow $\mathrm{F}$ denotes the force acting on the magnet system. each other downstream (five characteristic sizes of flow) using an iron yoke on. In this case, generated Lorentz force attains a value of $10 \mathrm{~N}$. The data in Figure 4 demonstrate the value of the force two times smaller than in Reference 5. The force acting on the magnet system is a linearly increasing function of the flow rate, as anticipated (Eq. [3]). Linearity of force is the result of flow rate $q$ constancy (i.e., at a decrease of flow cross section, a proportional increase of velocity takes place). It is true if the magnetic field of the LFF accomplishes a high vertical homogeneity and covers the whole height of flow. This circumstance is important to designing a LFF intended for the measurements in the closed channels because it is desirable for a LFF with an output independent of the shape of the velocity profile. At first glance, in practice, one would expect that the influence of the incoming profile would be significant. It should be also noted the works, ${ }^{[19,20]}$ where regimes of a generation of the large flow structures influencing a Lorentz force, are studied.

The results of the direct measurements of force as a function of $F(q)$ shown in Figure 4 give a value of a force coefficient $k=F / q=9.612 \times 10^{3} \mathrm{~N} \mathrm{~s} / \mathrm{m}^{3}$ that should be put into Eq. [5] to compute the calibration factor $c$. Using the electrical conductivity $\sigma$ of the working fluid and the magnetic field induction $B_{0}$ listed in Tables I and II, we obtain the following numerical value:

$$
c=k / \sigma B_{0}^{2}
$$

for the calibration coefficient of our laboratory LFF. The numerical value is $c=1.605 \times 10^{-2} \mathrm{~m}$. This fact reflects an important scaling property of LFF because it defines a characteristic half-length of magnetic field nonhomogeneity in flow direction.

\section{B. Industrial Measurements}

In this subsection, we describe the measurements performed using an industrial LFF prototype shown in Figure 5 within two production processes in a plant of a secondary aluminum manufacturer. All measurements are conducted in an open channel $l$ intended to transport molten aluminum 2 from a rotary furnace. The channel made of a concrete is supported by a nonmagnetic steel frame. The LFF prototype comprises a V-shaped magnet system with an opening angle of $60 \mathrm{deg}$, a mechanical system based on a pendulum principle, electronic scales with an accuracy of $10^{-4} \mathrm{~N}$, and computer to plot the measured Lorentz force as a function of time.

The form and dimensions of the magnet system are in agreement with a geometry of the production channel (Figures 5(a) and (b)). Each magnet pole 3 consists of $16 \mathrm{NdFeB}$ magnets with sizes of $30 \times 30 \times 100 \mathrm{~mm}$. The poles are linked to each other by an iron yoke 4 . Each pole area facing the lateral channel wall is $a \times b=100 \times 260 \mathrm{~mm}$ (where $a$ coincides with aluminum flow direction). The thickness of the poles is $60 \mathrm{~mm}$. The distance between the low edges of the poles is $300 \mathrm{~mm}$, whereas the upper edges are $550 \mathrm{~mm}$ apart 


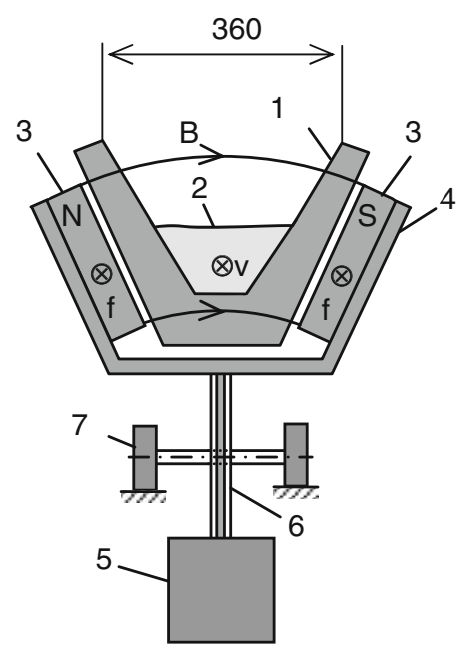

(a)

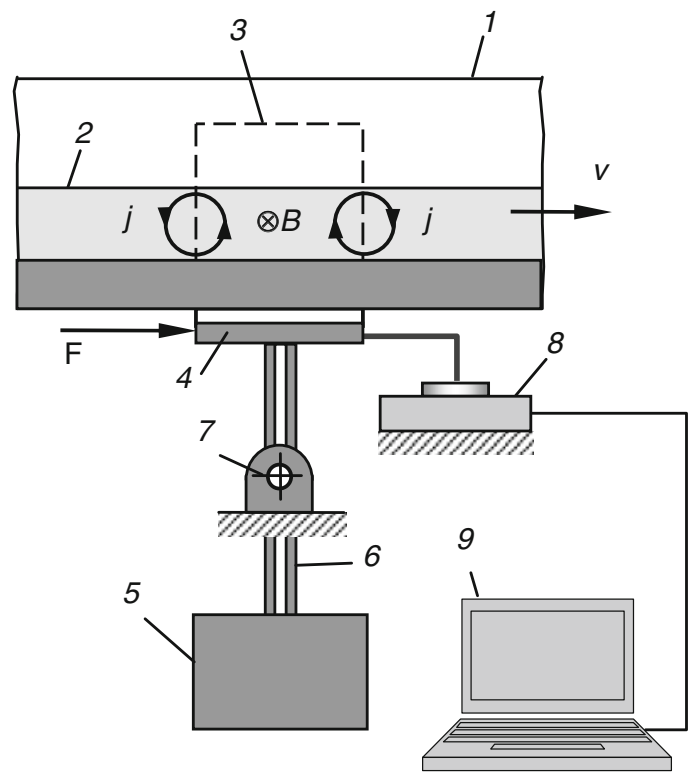

(b)

Fig. 5-Schematic of flow rate measurement in the industrial test of LFF. 1 is the channel, 2 is molten aluminum, 3 is the pole of permanent magnet, 4 is the iron yoke, 5 is the counterweight, 6 is the steel rod, 7 is the supporting axis, 8 is the digital scale, 9 is the computer, $\mathrm{B}$ is the magnetic field induction, $v$ is the molten flow velocity, $j$ is the electric current density, and F is the Lorentz force.

(Figure 5(a)). The magnetic induction on the magnet surface is $450 \mathrm{mT}$. The magnetic field $B$ at a height of $50 \mathrm{~mm}$ from the channel bottom in the middle plane is $13.5 \mathrm{mT}$. The magnet system is linked to a counterweight (5) by a steel rod (6) that can turn around the axis (7). The force $F$ is measured by commercial scales (8), and a digital signal from scales is fed to the computer (9) to compute the volumetric flow rate, mass flow rate, and accumulated volume and mass of aluminum.

The magnetic field in this LFF at first glance is represented as nonhomogeneous along the height for such a magnet system design. However, a special measurement of magnetic induction inside the channel directly before the industrial tests demonstrates a magnetic field homogeneous enough along the channel height of $0 \ldots 125 \mathrm{~mm}$ with a deviation of 1.13 pct. A height of flow can vary between $65 \mathrm{~mm}$ and $80 \mathrm{~mm}$. Such homogeneity is a result of the affinity of the horizontal part of the iron yoke to the lower edges of magnet poles as shown in Figure 5(a), which weakens this area transversal component of the magnetic field that provides equalization of the field along the vertical. In regard to the field distributions in two other directions, they are also identical for both industrial tests. Thus, for both tests used, factor $c$ reflects magnetic field nonhomogeneity in flow direction. In the industrial flow meter with a large distance between poles, the magnetic field is 30 times less than in the laboratory case. However, the much bigger flow rate in industrial tests takes place; therefore, this point provides a reliable measurement of Lorentz force in industry (Table II).

The positions of the main channel and the installed LFF from test to test do not vary. We emphasize that the channel and LFF are separated from each other with a gap of $10 \mathrm{~mm}$ between the magnet poles and the channel wall outer surface. The LFF is installed on a concrete foundation. Because of this, a channel position relative to LFF is rigorously fixed.

The weight of magnet system is $40 \mathrm{~kg}$. The counterweight, also about $40 \mathrm{~kg}$, provides equilibrium of the mechanical system. In the same manner as the laboratory experiment, we properly select a mass of counterweight to realize an initial unbalance of the mechanical system equal to $10^{-2} \mathrm{~N}$.

As shown in Figure 5(b) the eddy currents $j$ result in the vicinity of the edges of the magnetic field. Because the channel walls are nonconducting, the electric currents flow wholly inside the fluid. We emphasize that the aluminum oxide film on the upper surface of flow is motionless and plays a similar role as a nonconducting wall (i.e., eddy currents are fully closed in the liquid aluminum as sketched in Figure 5(b)). These currents are considerable as they are closed in the fluid in which the magnetic field is absent and an electromotive force is not generated. Therefore, the confined magnetic field provides a measurement of the Lorentz force by LFF at even a small induction of magnetic field.

The operating temperature of liquid aluminum in both tests is approximately $1053 \mathrm{~K}\left(780^{\circ} \mathrm{C}\right)$. Despite this, the temperature of the external surface of the channel walls near the LFF prototype does not exceed $323 \mathrm{~K}\left(50{ }^{\circ} \mathrm{C}\right)$; it makes cooling the magnet system unnecessary. To take into account the variation of the magnetic field induction in consequence of the magnet temperature changes (Eqs. [4] and [5]), we place a thermocouple on the magnet surface for a temperature correction of the processing data.

Two tests are executed. Test 1 is used as a base to define factor $c$ (Eq. [5]), using the known alloy mass received in this production cycle. It should be noted that this factor keeps a constant value if the magnetic field along the flow height changes within 1.13 pct (see previous sections). In the case of growth of the flow rate, an increase in the top level of flow adds a force according to Eq. [3]. 
We note that, in another case of nonhomogeneous magnetic field along the height, lifting of the flow level reaches an area of weaker magnetic field and gives a smaller increase in the Lorentz force (i.e., the factor $c$ seems underestimated). Therefore, we give a special attention to the vertical distribution of the magnetic field, the precise position of LFF, and inspection of the upper levels of the aluminum flows in the operating channel at a distance of the LFF installation $(3.5 \mathrm{~m}$ from the channel inlet) in the beginning and during both tests. It is important because, each time after aluminum transportation, the position of the channel outlet, which influences the height level of flow in the channel, is technically compelled to be disassembled for cleaning and reconditioning between the two transportation processes.

The results of the measurements are shown in Figure 6 where we plot the force acting on the LFF as a function of time. In both experiments reported, similar amounts of aluminum are discharged. Measurement 1 is characterized by a slow discharge, whereas in measurement 2 , the liquid metal is discharged with a higher flow rate. It is clearly shown that the higher flow rate in curve 2 results in a higher Lorentz force than in curve 1 . Moreover, the surfaces under curves 1 and 2 visually appear virtually equal in agreement with the fact that the cumulative masses discharged in tests 1 and 2 are similar.

To convert the measured forces into volumetric flow rates or mass flow rates, it is necessary to know the calibration constant $c$ for the LFF used in the plant tests. An accurate determination of this quantity would require an independent possibility to measure the instantaneous volumetric flow rate in the plant test. Because such a possibility does not exist, we had to determine the calibration constant in an indirect manner as is described next.

Strictly speaking, the calibration Eq. [3] is only valid if the cross section of the flow is constant; in which case, the flow rate $q$ is related to the mean velocity $v$ and the cross section $A$ by $q=v A$, where $A$ is a constant. In the open channel flow typical of secondary aluminum production, however, the cross section changes because of level fluctuations of the liquid metal. Hence, the calibration constant weakly depends on the level of the

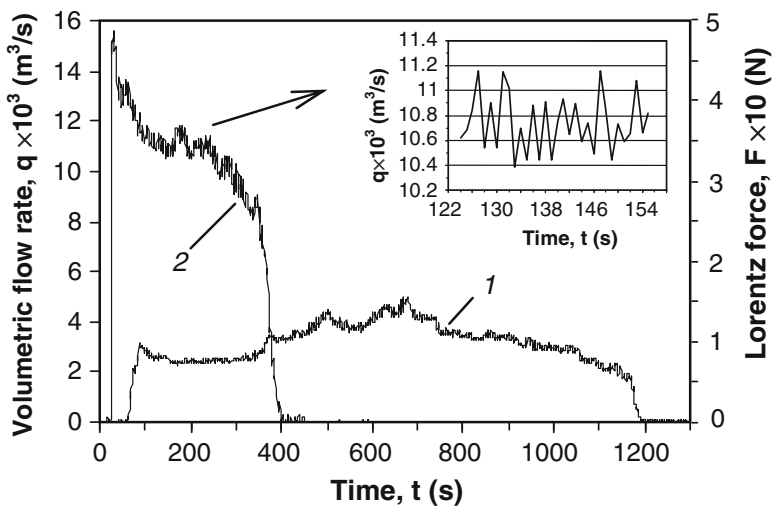

Fig. 6-Lorentz force and flow rate for two tests in industry as the functions of time; curves 1 and 2 correspond to tests 1 and 2, respectively; fragment of typical flow rate pulsations in test 2 is presented in the inset. liquid metal and thereby on $q$. In what follows, we assume that this dependence is so weak that $c$ can be regarded as a constant. We then can determine $c$ from the cumulative mass or volume.

The procedure is as follows. We carry out the measurements of Lorentz force $F$ within the process shown in Figure 6, curve 1, and compute an average value $F_{\text {av }}$ during a process time $\Delta t=1119$ seconds by integrating $F(t)$. We find that $F_{\mathrm{av}}=8.26 \times 10^{-2} \mathrm{~N}$. From weighing the discharged aluminum, we know its total mass $M=8350 \mathrm{~kg}$. From these two quantities and the tabulated value $\rho=2370$ (Table I) for the density, we obtain the average volume flow rate as follows:

$$
q=\frac{M}{\rho \Delta t}
$$

where $q=3.148 \times 10^{-3} \mathrm{~m}^{3} / \mathrm{s}$. In the same manner as the laboratory experiment, we calculate a force coefficient $k=F_{\mathrm{av}} / \mathrm{q}=26.24 \mathrm{~N} \mathrm{~s} / \mathrm{m}^{3}$. Following Eq. [15], we use the values of $k$, of the electrical conductivity $\sigma$, and of magnetic field induction $B$ from Table I to obtain the desired calibration factor as follows:

$$
c=4.78 \times 10^{-2} \mathrm{~m}
$$

This figure for $c$ is received through the direct measurement of alloy weight within basic test 1 and now is the characteristic of our LFF, and we apply it for the next measurements of flow in test 2 . In fact, the flow meter in this position becomes an instrument for measurement in the next cycles of aluminum flow rate. Curve 2 in Figure 6 is a result of the measurement of volumetric flow rate in test 2 by using of obtained factor $\mathrm{c}$ written in Eq. [17].

Curves 1 and 2 in Figure 6 distinctly indicate the beginning and end of two processes with a good accuracy. In the beginning of test 2, a sharp maximum resulting from a sudden flow from the furnace is observed. Fluctuations of flow feeding from the furnace generate a fluctuating force measured by LFF. Furthermore, the flow is turbulent, which is observed by inspecting the industrial Reynolds number given in Table II. We note that the LFF also measures the largescale perturbations in the flow with a frequency in the range of 0.3 to $0.6 \mathrm{~Hz}$ as shown by the inset in Figure 6 . Mechanical channel vibrations are excluded because of the special support of the LFF (operating channel and LFF are not connected mechanically). Therefore, the cause of such fluctuations can be linked to large-scale intensive turbulent pulsations generated by jet flow that comes into the channel from the furnace. The oscillations of a free surface of flow can serve as an additional contribution to fluctuations. However, the mean force signal directly indicates the mean flow rate. The ratio of amplitude of force oscillated by large-scale turbulent pulsations to a mean force $f^{\prime} / \mathrm{F}$ is less than 5 pct. Slow fluctuations of the mean force during the process sometimes can reach 12 pct (see both curves in Figure 6). We note that observed fluctuations play no essential role in receiving an accumulated mass during the process (Figures 7 and 8). 


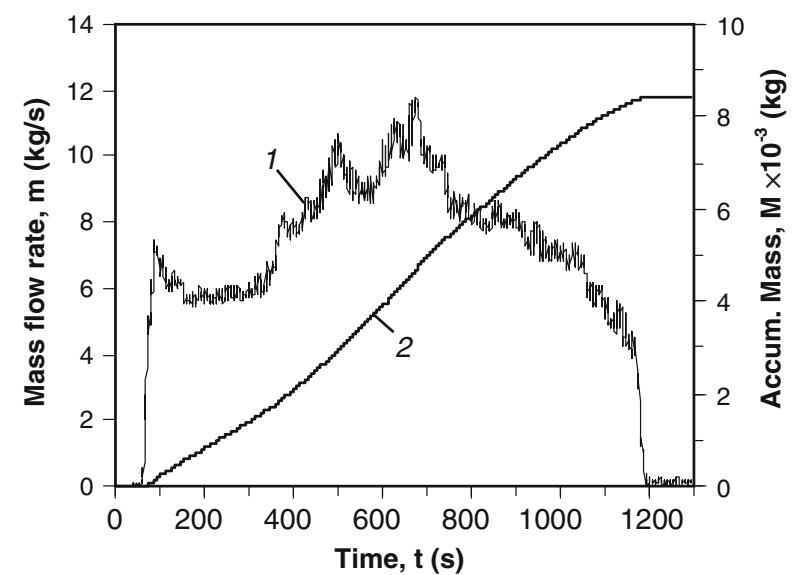

Fig. 7-Mass flow rate (curve 1) and accumulated mass (curve 2) obtained in the test 1 as a function of time.

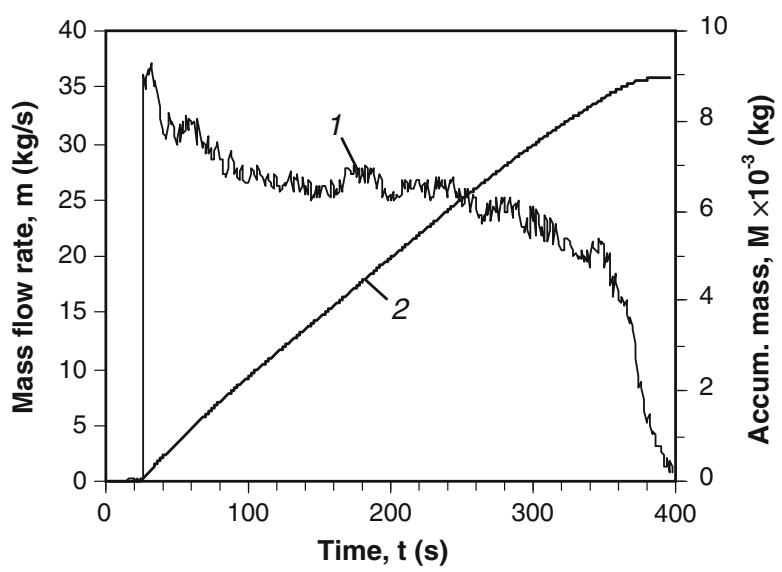

Fig. 8-Mass flow rate (curve 1) and accumulated mass (curve 2) obtained in the test 2 as a function of time.

To show the role of natural oscillations of LFF and channel in pulsating signal, we estimate their frequency. The magnet system with an arm length of $L=0.35 \mathrm{~m}$ and a mass of $M=80 \mathrm{~kg}$ possesses an inertia moment $I=10.17 \mathrm{~kg} \mathrm{~m}^{2}$ that leads to a natural frequency of oscillations $f^{\prime}{ }_{0 / \mathrm{mag}}=1.17 \mathrm{~Hz}$. These oscillations do not coincide with measured oscillations (Figure 6). Estimations of inertia moment for the channel of $M=1250 \mathrm{~kg}$ in mass and $L=10 \mathrm{~m}$ in length give a value of inertia moment of $I=5218 \mathrm{~kg} \mathrm{~m}^{2}$. These parameters provide the natural frequency of oscillations $f^{\prime} 0 / \mathrm{ch}=0.55 \mathrm{~Hz}$. This last frequency coincides in value with the legitimated frequency but is related to the channel oscillations in the vertical direction. In this connection, it should be noted that the LFF measures streamwise velocity oscillations and the mean velocity in this direction (i.e., does not measure oscillations in vertical direction).

In addition to the raw data shown in Figure 6 by curves 1 and 2, Figures 7 and 8 show the instantaneous mass flow rates $\mathrm{m}(\mathrm{t})$ (labeled 1 ) and cumulative mass $M$ (labeled 2) calculated according to Eqs. [8] and [9]. For the calculation, we use the density for alloy AlSil2 given in Table I. The curve 2 of the cumulative mass grows with time. The final value $M(\Delta t=1200$ seconds $)$ provides the total amount of aluminum that is passed through the channel. Curve 2 has the highest slope in the range between $t_{1}=400$ seconds and $t_{2}=650$ seconds in which the flow rate is maximum. In this test, the accumulated mass $M$ adds up to $8350 \mathrm{~kg}$.

Figure 8 demonstrates the dependences for test 2 as the same for test 1 in Figure 7. This process distinctive by its duration is also represented in the terms of mass flow rate (curve 1) and cumulative mass (curve 2) as functions of time. Curve 1 faithfully reproduces the behavior of curve 2 for the flow rate shown in Figure 6 . In the construction of curves in Figure 6 and Figure 8, we use Eqs. [6], [8,] and [9] as well as calibration factor $c$ (Eq. [17]). We emphasize that $q$ here at a smaller process duration is threefold higher than $q$ for test 1 . The accumulated mass reaches $8930 \mathrm{~kg}$.

It should be emphasized that the calibration factor $c$, whose dimension is meter, approximately seems to be half the length of the magnet system poles in the flow direction both for the laboratory and for the industrial LFF systems.

\section{Error Estimations of Industrial Measurements}

We perform the estimations of relative error of measurements for industrial tests. A correction of the magnetic field induction with a temperature variation is not conducted by virtue of the fact that temperature changes in a small range during the transportation process. This variation is approximately $280 \mathrm{~K}\left(7^{\circ} \mathrm{C}\right)$. Accounting for a change in the magnetic field with a temperature reported in this work, we find that error is $\left|\Delta_{\mathrm{B}(\mathrm{T})}\right|=0.85$ pct. Measurements of the Lorentz force performed by scales have an error of $\left|\Delta_{\mathrm{F}}\right|=0.05$ pct. Taking into account a possible variation of the molten alloy temperature of $303 \mathrm{~K}\left(30^{\circ} \mathrm{C}\right)$-relatively operating temperature $T=1053 \mathrm{~K}\left(780^{\circ} \mathrm{C}\right)$-we estimate the error of alloy electrical conductivity as $\left|\Delta_{\sigma(\mathrm{T})}\right|=$ $0.92 \mathrm{pct}$. The change in alloy temperature at $303 \mathrm{~K}$ $\left(30{ }^{\circ} \mathrm{C}\right)$ leads to alloy density variation. An estimation of error for the density of the given alloy is $\left|\Delta_{\rho(\mathrm{T})}\right|=$ 0.37 pct. Control measurement of weight (mass) in the basic test 1 with an accuracy of $10 \mathrm{~kg}$ gives an error of $\left|\Delta_{\mathrm{M}}\right|=0.15 \mathrm{pct}$. Thus, the relative error of flow rate measurement by our LFF is expressed as follows:

$|\xi|=\left|\Delta_{\mathrm{B}(\mathrm{T})}\right|+\left|\Delta_{\mathrm{F}}\right|+\left|\Delta_{\sigma(\mathrm{T})}\right|+\left|\Delta_{\rho(\mathrm{T})}\right|+\left|\Delta_{\mathrm{M}}\right|=2.34 \mathrm{pct}$

This estimation can be reduced to $|\xi|=0.57$ pct if introducing a correction of magnetic field induction (Eq. [4]) by measuring the temperature of the magnet system, and the correction of electrical conductivity can be reduced by measuring the molten alloy temperature at times, using corresponding software.

\section{CONCLUSIONS}

We have described a noncontact measurement method for the velocity of electrically conducting fluids and demonstrated its feasibility for measuring flow rates. Laboratory experiment and industrial tests showed that 
the LFF is a reliable tool for the measurement of flow characteristics of aggressive molten metal even for a small magnitude of the magnetohydrodynamics interaction parameter.

Our measurements in the laboratory show that a linear dependence between the Lorentz force and the flow rate holds for a closed channel. Two industrial tests also demonstrate the feasibility of measuring the flow characteristics in open channels.

The results serve as a basis for the development of new LFF systems with improved performance. Systems currently under construction have parallel magnet poles and have a higher magnetic field. This is not only important for the measurements in aluminum but also for molten metals with a lower electrical conductivity such as steel, lead, and tin. Other applications besides metallurgy such as semiconductor crystal growth and glass manufacturing are forthcoming.

\section{ACKNOWLEDGMENTS}

We are grateful to the Bundesministerium für Bildung und Forschung (BMBF) for partial financial support in the framework of the ForMaT program under grant number 03FO2202. Moreover, H. Schreiber and D. van Kalken are gratefully acknowledged for useful discussions.

\section{OPEN ACCESS}

This article is distributed under the terms of the Creative Commons Attribution Noncommercial License which permits any noncommercial use, distribution, and reproduction in any medium, provided the original author(s) and source are credited.

\section{REFERENCES}

1. M.E. Schlesinger: Aluminum Recycling, CRC Press, Boca Raton, FL, 2006.

2. A.R. Khoei, I. Masters, and D.T. Gethin: J. Mater. Process. Tech., 2002, vol. 127, pp. 96-106.

3. C. Karcher, H. Schreiber, and Y. Kolesnikov: Proc. Int. Workshop Electroheat, Ilmenau, Germany, 2005.

4. A. Thess, Y. Kolesnikov, C. Karcher, and E. Votyakov: Proc. 5th Int. Symp. Electromagnetic Processing Materials, Sendai, Japan, 2006, pp. 731-34.

5. A. Thess, E. Votyakov, and Y. Kolesnikov: Phys. Rev. Lett., 2006, vol. 96, pp. 4501-05.

6. A. Thess, Y. Kolesnikov, and C. Karcher: Patent WO 2007/ $033982,2007$.

7. Y. Kolesnikov and A. Thess: Proc. 6th Int. Symp. Electromag. Processing Materials, Ilmenau, Germany, 2009, pp. 41-44.

8. C. Karcher, Y. Kolesnikov, and A. Thess: Patent DE 102007038 $685,2008$.

9. A. Thess, Yu. Kolesnikov, C. Karcher, and V. Minchenya: Proc. 6th Int. Symp. Electromag. Processing Materials, Ilmenau, Germany, 2009, pp. 379-82.

10. C. Karcher, Y. Kolesnikov, V. Minchenya, and A. Thess: Proc. 6th Int. Symp. Electromag. Processing Materials, Ilmenau, Germany, 2009, pp. 419-22.

11. P.A. Davidson: Annu. Rev. Fluid Mech., 1999, vol. 31, pp. 273-300.

12. M.G. Abele: Structures of Permanent Magnets, Wiley, New York, NY, 1993.

13. M. Sagawa, S. Fujimura, H. Yamamoto, Y. Matsuura, and S. Hirosawa: J. Appl. Phys., 1985, vol. 57, p. 4094.

14. J.A. Shercliff: The Theory of Electromagnetic Flow Measurement, Cambridge University Press, Cambridge, U.K, 1961.

15. R. Brandt and G. Neuer: Int. J. Thermophys., 2007, vol. 28, pp. 1429-46.

16. J. Knebel and L. Krebs: Exp. Therm Fluid Sci., 1994, vol. 8, pp. $133-48$.

17. O. Andreev, Y. Kolesnikov, and A. Thess: Exp. Fluid, 2009, vol. 46, pp. 77-83.

18. O. Andreev, Y. Kolesnikov, and A. Thess: Phys. Fluids, 2007, vol. 19, p. 9902.

19. E.V. Votyakov, Y. Kolesnikov, O. Andreev, E. Zienicke, and A. Thess: Phys. Rev. Lett., 2007, vol. 98, p. 4504.

20. E.V. Votyakov, E. Zienicke, and Y.B. Kolesnikov: J. Fluid Mech., 2008, vol. 610, pp. 131-156. 